Meta

Journal des tradlucteurs

Translators' Journal

\title{
ATA - Translation: Theory and Practice - Tension and Interdependance (1991) : ATA Scholarly Monograph Series, Volume V, State University of New York at Binhamton (SUNY), $270 \mathrm{p}$.
}

\section{Georges L. Bastin}

Volume 38, numéro 2, juin 1993

URI : https://id.erudit.org/iderudit/001996ar

DOI : https://doi.org/10.7202/001996ar

Aller au sommaire du numéro

Éditeur(s)

Les Presses de l'Université de Montréal

\section{ISSN}

0026-0452 (imprimé)

1492-1421 (numérique)

Découvrir la revue

Citer ce compte rendu

Bastin, G. L. (1993). Compte rendu de [ATA — Translation : Theory and Practice - Tension and Interdependance (1991) : ATA Scholarly Monograph Series, Volume V, State University of New York at Binhamton (SUNY), 270 p.] Meta, 38(2), 365-366. https://doi.org/10.7202/001996ar d'utilisation que vous pouvez consulter en ligne. 
ATA - Translation: Theory and Practice - Tension and Interdependence (1991): ATA Scholarly Monograph Series, Volume V, State University of New York at Binhamton (SUNY), $270 \mathrm{p}$.

Il y en a pour tous les goûts et toutes les hantises dans ce cinquième volume des séries ATA. Le thème choisi, théorie $v s$ pratique, se prête évidemment aux réflexions les plus variées. L'éventail des auteurs (Australie, Chine, Israël et Brésil en plus de l'Europe et de l'Amérique du Nord) est certes large (19 en tout), mais l'éditeur, Mildred L. Larson, parvient à les regrouper en six sous-thèmes: le processus de traduction, aspects théoriques, la traduction de genres (en dehors de la prose), la mise en pratique de la théorie, questions linguistiques spécifiques, la traduction à l'université. Tout un programme! Que nous ne suivrons pas pour rendre compte de l'ouvrage.

Question de goût, les amateurs de poésie, d'opéra, de théâtre et d'humour sont bien servis par B. Raffel, M. Herman \& R. Apter, A. Brisset et H. Niedzielski respectivement. Le premier, par l'examen de bonnes et mauvaises traductions de textes poétiques indiens et indonésiens, souligne, parmi les aptitudes indispensables au traducteur, le sens poétique de celui-ci et met en lumière cinq principes régissant la traduction poétique. Les seconds démontrent qu'en dépit des contraintes traduire un opéra est chose possible et souhaitable. La troisième défend avec passion et brio le Macbeth québécois de M. Garneau et le dernier illustre comment une «analyse componentielle» permet de surmonter les obstacles culturels de la traduction de l'humour.

Question de goût encore, on lira avec intérêt le point de vue des théoriciens soviétiques concernant la notion de temps et d'espace (L. G. Leighton), la vision chinoise des critères de qualité et des méthodes de traduction (Fan Shouyi), l'histoire récente de la politique éditoriale et des normes correspondantes en matière de traduction en Isrä̈l (R. Weissbrod), et la raison et la justification de la liberté d'altérer les textes japonais pour les rendre accessibles au lecteur anglophone (J. Wakabayashi).

Question de hantise, on retrouve les sempiternels débats sur la dichotomie traduction littérale/traduction libérale, sur l'évaluation, sur la notion d'équivalence et sur le besoin d'adapter. La première question est largement débattue par S. Viaggio, mais aussi par M. Scott Doyle qui traduit sous forme de schémas «l'entreprise» traductionnelle et les forces centripède et centrifuge agissantes. $M$. Gaddis Rose et $H$. Gerzymisch-Arbogast \& U. Schnatmeyer analysent, la première dans une optique de praticienne, les autres sous l'angle pédagogique, le processus de traduction en tant qu'activité non neutre, en tant que contrat à remplir. L'évaluation de la qualité est au cceur des articles de D. Gile et de E. W. Deibler. Gile, se référant tant à la traduction qu'à l'interprétation, définit la traduction comme un acte de communication et examine les tenants et les aboutissants de cette définition en rapport avec la qualité. Deibler suggère aux traducteurs d'avoir l'humilité de soumettre leur travail au regard vérificateur de «native» lecteurs. Quant à l'équivalence, traitée de près ou de loin dans tous les articles, elle acquiert une dimension neuve chez $R$. Rabadán, qui fonde son analyse de l'unité de traduction («unité de pensée», «logèmes», «inforème» et «translème») sur le modèle d'équivalence choisi par le traducteur. Enfin, nombreux sont les articles insistant sur le besoin d'adapter sans toutefois oser utiliser le terme «adaptation» (exception faite de Niedzielski dans son travail sur l'humour). Outre ce dernier, les exemples les plus éloquents sont ceux d'A. Brisset et son Shakespeare dans une "nation ben misérabe», et de J. Wakabayashi, confrontée à la traduction anglaise des paragraphes d'introduction et de conclusion de textes japonais.

Ce cinquième volume comporte aussi un volet polémique, représenté par trois règlements de comptes. Le premier (en fait le dernier), celui de F. Gomes De Matos, à l'égard de la traduction didactique. L'auteur s'élève violemment contre l'interdiction de 
traduire en classe de langue et revendique pour la traduction didactique le statut de «droit de l'homme» et une mission universelle de paix. Sergio Viaggio, quant à lui, s'en prend, avec une ironie certaine, à $\mathrm{P}$. Newmark et à son «We do translate words, because there is nothing else to translate : [...]» (Newmark 1988: 73). Rejetant l'opposition newmarkienne entre «traduction sémantique» et «traduction communicative», Viaggio prône une méthode unique, à savoir «the translator makes the right extralinguistic sense the right linguistic way» (p. 177), qu'il illustre judicieusement par la traduction de deux extraits, l'un poétique, l'autre prosaïque. Le dernier règlement de comptes, et loin d'être le moindre, revient à J.-P. Vinay. D'abord à l'égard des Américains à qui il reproche de négliger la littérature traductologique francophone. Ensuite avec la théorie et les théoriciens qui, sauf quelques exceptions, se placent hors de portée des praticiens. Vinay prêche pour une traductologie appliquée, pour une théorie au service de la pratique, bref quelque chose dans le genre de... la SCFA. Et gare à ceux qui osent la remettre en question! Selon Vinay, il y a dans le processus de traduction une part significative d'automatismes propres à être enseignés; telle serait la tâche de la théorie. L'article se termine sur des exemples «difficiles» et quelques trouvailles.

Pour être complet, il nous faut encore mentionner un article qui ne fera certes pas la joie de J.-P. Vinay, mais qui a un intérêt linguistique théorique certain : la catégorisation naturelle du lexique et l'utilité d'un «modèle grammatical à deux cycles» de M. Thelen. Pour clore, un article exclusivement consacré à la métaphore, aux métaphores décrivant le processus de traduction. On passe ainsi du filet de pêche, aux jeux de construction pour en arriver aux différentes façons de couper la viande et à l'illusion créée par les acteurs de théâtre. Intéressant.

GEORGES L. BASTIN 